\title{
Dyslexia: reconciling controversies within an integrative developmental perspective
}

\author{
Bart Boets ${ }^{1,2}$ \\ ${ }^{1}$ Child and Adolescent Psychiatry, KU Leuven, Leuven, Belgium \\ ${ }^{2}$ Department of Brain and Cognitive Sciences and McGovern Institute for Brain Research, Massachusetts Institute of Technology \\ (MIT), Cambridge, MA USA
}

The predominant phonological deficit hypothesis of dyslexia has been challenged by converging neural and behavioral evidence for intact but less-accessible speech sound representations in adult dyslexic readers. I propose here that recognizing the dysfunctional connection between left frontal and temporal language regions as the primary hallmark of dyslexia could explain the degraded phonological representations in young children with dyslexia, as well as the intact but less-accessible phonological representations in mature dyslexic individuals.

Developmental dyslexia is a hereditary neurological disorder characterized by severe and persistent reading and/or spelling impairments, despite normal intelligence and adequate schooling. It is well established that individuals with dyslexia perform poorly on tasks that require phonological awareness, verbal short-term memory and speeded lexical retrieval, and that performance on these high-level phonological tasks is associated with literacy acquisition in both typical and dyslexic readers [1]. Most researchers in this field assume that the common variance among these tasks reflects the quality of underlying phonological representations (although some consider lexical retrieval as a distinct ability; $c f$. the so-called double-deficit hypothesis). Accordingly, the most prominent and parsimonious dyslexia account postulates that speech sound representations are somehow degraded or less well specified in individuals with dyslexia, and that this primary representational deficit impacts upon higher-level phonological processing and reading development. Corroborating evidence for this hypothesis has been put forward by studies showing impaired speech perception, in particular in young children with dyslexia. By extension, in light of the (mixed) evidence for auditory processing and speechperception impairments in dyslexia, a causal pathway has been proposed - wherein basic auditory problems interfere with accurate speech perception and subsequently hamper the development of accurate phonological representations [2].

However, the leading phonological deficit hypothesis on dyslexia has recently been challenged by studies asserting that the phonological representations per se may be intact in individuals with dyslexia, but the ability to access them is impaired. Ramus and colleagues reached this conclusion based on a series of in-depth cognitive studies in adults

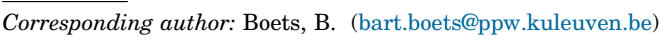

1364-6613/

(c) 2014 Elsevier Ltd. All rights reserved. http://dx.doi.org/10.1016/j.tics.2014.06.003 with dyslexia and a thorough review of the available literature [3]. More recently, Boets et al. provided neural evidence that phonological representations in adult dyslexic readers are exactly as robust and distinct as those in typical readers, but are less accessible for higher-order phonological processing [4]. Although both sets of studies convincingly converge on the conclusion that the phonological representations per se are intact, these new findings cause some tension in the field because they oppose the predominant view on dyslexia over the past 40 years. A relevant detail, however, is that both studies demonstrating intact phonological representations have been conducted in adults with dyslexia, whereas the bulk of electrophysiological and speech-perception studies supporting the degraded representations hypothesis have been conducted in young children with dyslexia or even pre-readers at risk of dyslexia (e.g., the longitudinal study by Molfese [5], the Jyväskylä Longitudinal Study of Dyslexia, and the Dutch Dyslexia Program). Thus, it remains to be seen to what extent these new findings in adults can be generalized to a child population. I propose here some suggestions towards reconciling these seemingly conflicting findings within an integrative developmental perspective.

Two major possibilities are envisaged. The more drastic posits that a more fine-grained neural and cognitive investigation will also reveal that, in children with dyslexia, the phonological representations are exactly as sharp as in their typically reading peers. Hence, analogously to the adult findings [3,4], phonological deficits would only occur in dyslexic children as a result of particular task demands such as short-term memory, conscious attention and time constraints, and they will not appear in the more basic speech-processing measures. Although certainly controversial, such a conclusion is not implausible given the particularly mixed evidence within this field. However, in view of the extensive evidence suggesting that children with dyslexia process speech sounds with lower fidelity $[5,6]$, this position might ultimately not be tenable.

A second and more intermediate perspective proceeds from the growing evidence for a dysfunctional fronto-temporal connection in dyslexia, which has been interpreted as neural evidence for impaired access to phonological representations [4]. There is reason to believe that this particular disconnection may be a primary hallmark of dyslexia because its neuroanatomical substrate (i.e., the left arcuate fasciculus) has consistently been shown to be impaired in dyslexic individuals [7], even in preschool pre-readers at risk of dyslexia [8]. This major white-matter language tract 


\section{Box 1. Structural brain connectivity: the left arcuate} fasciculus

The left arcuate fasciculus (see Figure 1 in main text) is a major white-matter language tract and connects Wernicke's region in left posterior superior temporal gyrus with the premotor cortex in precentral gyrus and Broca's region in left inferior frontal gyrus. Wernicke's region is involved in speech perception and hosts the phonological representations; the premotor cortex and Broca's region are involved in speech production, subvocal rehearsal, sensory-motor integration, and higher-level phonological processing $[4,9,11]$. Diffusion tensor imaging (DTI) and diffusion weighted imaging (DWI) are non-invasive MRI techniques providing in vivo information about the structural properties and orientation of whitematter fiber tracts in the brain. They offer an index of white-matter integrity through indirect measures of myelination, axon density and caliber, and directional coherence of axons. Several studies have shown that the left arcuate fasciculus is involved in phonological processing and the indirect phonological reading route, and that its integrity is impaired in dyslexic readers $[4,7]$ and in young pre-readers at risk of dyslexia because of poor phonological skill [8]. A comprehensive review and quantitative meta-analysis of DTI studies also identified this white-matter tract as the major anatomical substrate involved in reading and dyslexia [7]. Recently, reinforcement of this anatomical connection was shown in exilliterates who learned to read, hence suggesting plasticity of the arcuate fasciculus in response to reading experience [15].

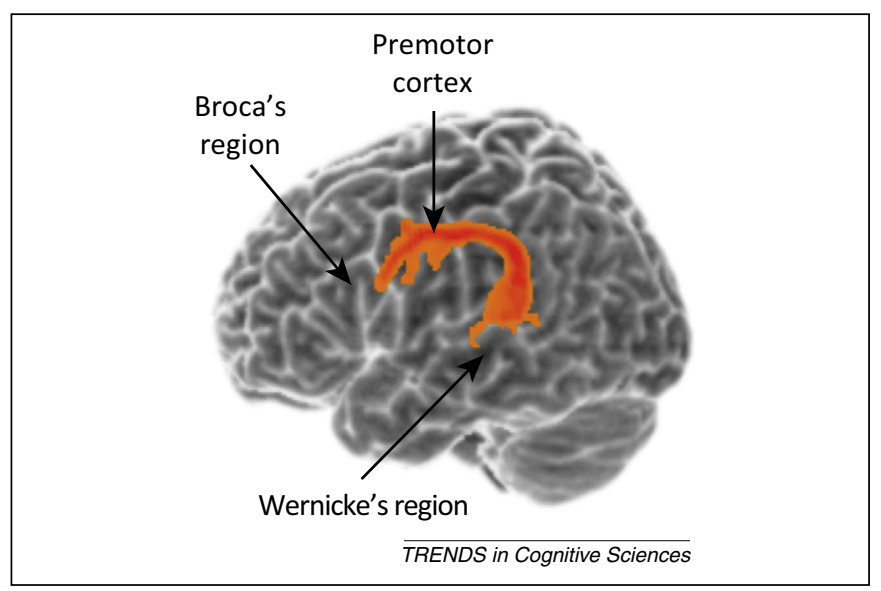

Figure 1. Left arcuate fasciculus on a standard rendered brain.

connects the auditory cortex with the premotor cortex and the left inferior frontal gyrus, hence constituting the primary interface between sensory and articulatory speech processing [9] (Box 1). Phonological representations are not inbuilt in newborns but gradually emerge through exposure to the natural language environment. By spontaneously extracting the statistical regularities that are informative in a particular language, the infant brain becomes tuned to the language-specific speech sounds or phonemes [10]. Given that the development of phonological representations requires an intensive interaction between the auditory system and the oromotor system [11], impaired functional and structural connection between the two systems may very well slow down this development (Boxes 1 and 2). Accordingly, we may expect underdeveloped phonological representations in young children with dyslexia, as evidenced for instance by deficient speech-perception performance [5,6]. However, as has been reported in the cognitive literature [3], and as confirmed at the neural level [4], these initial representational

\section{Box 2. Functional brain connectivity}

Even when at rest, the brain shows a continuous pattern of slowly fluctuating background activity. These fluctuations occur not randomly, but represent neural activity organized into structured spatiotemporal profiles that constitute the functional architecture of the brain. Functional connectivity is defined as a correlation between remote neurophysiological events in the temporal domain, and can be derived from resting-state and task-evoked functional MRI data. Separate anatomical regions with similar functional properties tend to exhibit coherent fluctuations in spontaneous activity, believed to reflect (the history of) functional communication between them. They constitute networks which are prepared to carry out common tasks. A recent study [4] investigated the functional connectivity between 12 brain regions involved in auditory processing, speech perception, and higher-level phonology, and showed - very specifically - that the functional communication between Broca's region and left and right superior temporal gyrus was impaired in dyslexic readers. Moreover, individual differences in the strength of functional connectivity between Broca's and Wernicke's region were highly significantly associated with various behavioral indices of word reading and phonology.

problems resolve over time (perhaps as a consequence of reading instruction or remedial training), and dyslexics ultimately present intact phonological representations which are as robust and distinct as those of typical readers. Nevertheless, what is left is the dysfunctional connection between the frontal and temporal language regions, which behaviorally manifests itself as a deficit in access to otherwise intact phonological representations, and which is evident in tasks requiring higher-level phonological processing such as conscious manipulation, speeded retrieval or short-term memory of speech sounds. Notably, a recent longitudinal study neatly confirmed this predicted developmental pattern by demonstrating impaired speech-innoise perception and impaired higher-level phonological processing (thus deficient representations and deficient access) in preschoolers that later develop dyslexia, but intact speech-in-noise perception and impaired higherlevel phonological skills (indicative of intact representations and deficient access) when these same children attended first grade of primary school [2]. Because learning to read and write requires not only intact phonological representations but also conscious and fluent access to these representations, these children are at risk of developing reading problems. Indeed, the continuous speech stream has to be segmented in individual speech sounds to map them on their corresponding graphemes, and the phonemes must be blended together in short-term memory to decode the word. Hence, the fragile access to the phonological structure of language will hamper the acquisition of literacy.

It is noteworthy that particular task and stimulus characteristics specifically recruit this left fronto-temporal connection. Tasks involving verbal short-term memory, for instance, activate the left inferior frontal gyrus and depend on the cycling of information between a phonological buffer involved in auditory perception (situated in Wernicke's area) and one involved in speech production (situated in Broca's area) [9]. Likewise, there is evidence that effortful speech processing (e.g., categorical perception of a speech continuum) [9], and even basic auditory (temporal) processing [12], mobilize this left frontal brain region, which is 
likely to access the sensory trace of the auditory signal through the same fronto-temporal connections that have been shown to be deficient in dyslexia. Therefore, it is plausible that some of the observed deficits in dyslexia are secondary consequences of using task designs and stimuli that require efficient communication within an intact left fronto-temporal circuit.

It may be noticed that the proposed account of dyslexia is reminiscent of the traditional conceptualization of conduction aphasia as a fronto-temporal disconnection, and one may wonder why dyslexic individuals do not present more prominent speech production deficits. An answer can be found in recent advances in neuroimaging that provide evidence for two parallel dorsal arcuate pathways, one from the temporal cortex to the premotor cortex and one from the temporal cortex to the inferior frontal gyrus (Broca's area or BA 44 pars opercularis), with the former mainly supporting sound-to-motor mapping and the latter supporting higher-level language processes [13]. In line with explanations of conduction aphasia as a type of speech apraxia, it has been suggested that this phenomenon primarily entails a dysfunctional connection with the premotor cortex which is involved in speech programming [14]. For dyslexia, however, a dysfunctional connection with Broca's region is envisaged as the principal bottleneck, hence primarily resulting in metalinguistic or higher-level phonological problems. Thus far, diffusion tensor imaging (DTI) studies in dyslexia have not yet disentangled these two dorsal tracts, but a functional connectivity study [4] did reveal specifically reduced communication between Broca's area and left and right superior temporal gyrus in individuals with dyslexia.

Taken together, a preliminary attempt towards reconciling seemingly conflicting findings in the dyslexia literature is provided here by consideration of the developmental consequences of a dysfunctional left fronto-temporal brain connection. Future empirical research will be necessary to test and validate these seminal ideas and to reveal which of the outlined options captures the developmental trajectory of children with dyslexia. In this regard, systematic investigation of the quality of phonological representations in individuals with dyslexia from infancy to adulthood is warranted, both through electrophysiological and in-depth behavioral studies. A comprehensive review and metaanalysis of dyslexia studies covering basic speech perception versus higher-order phonological processing may constitute an essential first step towards revealing whether problems at the representational level resolve over time whereas higher-order phonological problems persist. Future neuroimaging studies should also aim at elucidating the specificity of the deficient functional and structural connections with the frontal cortex in dyslexia.

\section{Acknowledgments}

B.B. is a postdoctoral research fellow of the Research Foundation Flanders (FWO). The research was financed by a Fulbright grant and a grant of the KU Leuven Research Council (IDO/10/003). I thank Franck Ramus and Maaike Vandermosten for fruitful discussion.

\section{References}

1 Wagner, R.K. and Torgesen, J.K. (1987) The nature of phonological processing and its causal role in the acquisition of reading skills. Psychol. Bull. 101, 192-212

2 Boets, B. et al. (2011) Preschool impairments in auditory processing and speech perception uniquely predict future reading problems. Res. Dev. Disabil. 32, 560-570

3 Ramus, F. and Szenkovits, G. (2008) What phonological deficit? Q. J. Exp. Psychol. (Colchester) 61, 129-141

4 Boets, B. et al. (2013) Intact but less accessible phonetic representations in adults with dyslexia. Science 342, 1251-1254

5 Molfese, D.L. (2000) Predicting dyslexia at 8 years of age using neonatal brain responses. Brain Lang. 72, 238-245

6 Hornickel, J. and Kraus, N. (2013) Unstable representation of sound: a biological marker of dyslexia. J. Neurosci. 33, 3500-3504

7 Vandermosten, M. et al. (2012) A qualitative and quantitative review of diffusion tensor imaging studies in reading and dyslexia. Neurosci. Biobehav. Rev. 36, 1532-1552

8 Saygin, Z.M. et al. (2013) Tracking the roots of reading ability: white matter volume and integrity correlate with phonological awareness in prereading and early-reading kindergarten children. J. Neurosci. 33, $13251-13258$

9 Hickok, G. et al. (2011) Sensorimotor integration in speech processing: computational basis and neural organization. Neuron 69, 407-422

10 Kuhl, P.K. et al. (1992) Linguistic experience alters phonetic perception in infants by 6 months of age. Science 255, 606-608

11 Schulze, K. et al. (2012) Test of a motor theory of long-term auditory memory. Proc. Natl. Acad. Sci. U.S.A 109, 7121-7125

12 Gaab, N. et al. (2007) Neural correlates of rapid auditory processing are disrupted in children with developmental dyslexia and ameliorated with training: an fMRI study. Restor. Neurol. Neurosci. 25, 295-310

13 Friederici, A.D. (2011) The brain basis of language processing: from structure to function. Physiol. Rev. 91, 1357-1392

14 Bernal, B. and Ardila, A. (2009) The role of the arcuate fasciculus in conduction aphasia. Brain 132, 2309-2316

15 Thiebaut de Schotten, M. et al. (2014) Learning to read improves the structure of the arcuate fasciculus. Cereb. Cortex 24, 989-995 